\title{
Ultrarelativistic outflows associated to progenitors of Gamma-Ray Bursts
}

\section{Miguel-Angel Aloy*}

Department for Astronomy and Astrophysics, University of Valencia

E-mail: miguel.a.aloy@uv.es

\section{Petar Mimica}

Department for Astronomy and Astrophysics, University of Valencia

E-mail: petar.mimica@uv.es

Gamma-ray bursts (GRBs) are nowadays considered to be collimated ejecta with bulk velocities very close to the speed of light. The mechanism by which after the quasy-isotropic energy release of a few $10^{50} \mathrm{erg}$ yields a collimated ejection of plasma has not been satisfactorily explained by simple analytically developments. The reason being that the collimation of an outflow by its progenitor system depends on a very complex and non-linear dynamics. That has made it necessary to use numerical simulations in order to shed some light on the viability of some likely progenitors of GRBs. Here we review the most relevant features shown by these numerical simulations and how they have been used to validate the most popular progenitor models, namely, the collapsar model (for long GRBs) and the model involving the merger of compact binaries (for short GRBs).

Supernovae: lights in the darkness

October 3-5, 2007

Maó (Menorca)

* Speaker. 


\section{Introduction}

Gamma-ray bursts (GRBs) are probably produced in the course of the birth of an accreting, stellar-mass black hole (BH). In other astrophysical systems where accreting BHs fuel collimated beams of plasma (e.g., AGNs and BH-X-ray binaries), collimated and relativistic jets are directly imaged. Thus, it is assumed that also GRBs result from relativistic, collimated outflows from accreting, stellar-mass BHs. We have inferred that outflows yielding GRBs are relativistic because of a couple of observational constraints. First, radio scintillation of the interstellar medium has been detected [16] and also superluminal proper motions have been seen in imaged afterglows [56]. The ultrarelativistic expansion is also necessary to overcome the theoretical constrain imposed by the compactness problem [12]. However, we only have an indirect evidence of collimation based on the observational constraint posed by the achromatic break in the afterglow light curve of some GRBs (e.g., [21]). From the theoretical point of view, if GRBs are collimated events, the true emitted energy $E_{\gamma}$ is reduced by a factor $f_{\Omega} \simeq \theta^{2} / 2$ [49, 54], i.e., $E_{\gamma}=f_{\Omega} E_{\gamma \text {,iso, }}$, where $E_{\gamma, \text { iso }}$ is the detected equivalent isotropic energy. Nonetheless, the mechanism by which after the quasi-isotropic release of an amount of energy in the range $10^{48}-10^{51}$ erg results in a collimated ejection has not satisfactorily been explained analytically. The reason being that the collimation of an outflow by the progenitor system depends on a very complex and non-linear dynamics. That has made necessary the use of numerical simulations in order to understand the collimation mechanism as well as to shed some light on the viability of some systems proposed to be the progenitors of GRBs. A robust result obtained in numerical simulations of generation of GRBs is that the progenitor system yields collimated outflows under rather general conditions independent on whether the outflow is initiated thermally (e.g., [4, 6]) or it is magnetically driven [31].

In this contribution I will review the most relevant features shown by these numerical simulations and how they have been used to validate the collapsar model (for long GRBs; § 2) and the model involving the merger of compact binaries (for short GRBs; § 3).

\section{Outflows emerging from progenitors of long GRBs}

Among the plethora of models devised to explain the origin of long GRBs (lGRBs), the most popular is the collapsar ${ }^{1}$ [57]. A collapsar results from the collapse of a massive $\left(M_{\text {ZAMS }} \sim 30 \mathrm{M}_{\odot}\right)$, fast-rotating $\left(3 \cdot 10^{16} \mathrm{~cm}^{2} \mathrm{~s}^{-1} \leq j \leq 2 \cdot 10^{17} \mathrm{~cm} \mathrm{~s}^{-1}\right)$ star that does not form a successful supernova but collapses to a $\mathrm{BH}\left(M_{\mathrm{BH}} \sim 3 \mathrm{M}_{\odot}\right)$ surrounded by a thick disk. The viscous accretion of the disk matter onto the $\mathrm{BH}$ yields a strong heating that, in its turn, produces a copious amount of thermal neutrinos and antineutrinos, which annihilate preferentially around the rotation axis producing a fireball of $e^{+} e^{-}$pairs and high energy photons. Later it was noted that, perhaps $v$-powered fireballs might not be sufficiently energetic to fuel the most powerful GRB events and, thus, the collapsar model was extended to account for alternative energy extraction mechanisms [29]. Most of these alternative mechanisms invoke the existence of strong magnetic fields to produce a Pointing flux dominated flow (see, e.g., [17]). More explicitly, the accretion energy of the torus could be tap by sufficiently strong magnetic fields (hydromagnetic generation) by means of the Blandford-Payne

\footnotetext{
${ }^{1}$ originally, the term failed supernova was coined to refer to this model
} 
process [11], or a non-trivial fraction of the rotational energy of the BH may also be converted into a Poynting flux [10].

The scape of the newly born fireball and its terminal Lorentz factor $\left(\Gamma_{\infty}\right)$ depend on structural and on dynamical factors. The critical structural factors are the environmental baryon density in the funnel around the rotation axis of the star and the ability of the progenitor star to loose its outer Hydrogen envelope. An under-dense funnel forms along with the accretion torus if the specific angular momentum of the core of the star lies in the range $3 \times 10^{16} \mathrm{~cm}^{2} \mathrm{~s}^{-1} \lesssim j \lesssim 2 \times 10^{17} \mathrm{~cm}^{2} \mathrm{~s}^{-1}$ [28]. The existence of the funnel is crucial for the collimation of the fireball and to permit its propagation through the progenitor. The most favourable conditions for the generation and propagation of the fireball happen when the density of the funnel $\left(\rho_{\mathrm{f}}\right)$ is much smaller than the density of the torus ( $\rho_{\text {torus }}$ ), namely, $\rho_{\mathrm{f}} / \rho_{\text {torus }} \lesssim 10^{-4}-10^{-3}$ [28]. The likelihood that the progenitor star had lost its Hydrogen envelope depends on a number of factors which occurrence is still a matter of debate like, e.g., the generation of stellar winds, the interaction with a companion [45], etc. The relevance of the loss of the hydrogen envelope resides on the fact that, unless the density of the funnel is extremely small (as proposed by [32]), only a mildly relativistic, poorly-collimated fireball would reach the outer edge of the hydrogen envelop after very long times and with relatively small Lorentz factors $(\Gamma \simeq 2)$ implying that the observational signature would be an $\mathrm{X}$-ray/UV transient with a duration of $\sim 100-1000$ s but not a GRB [29]. Finally, the most important dynamical factor setting $\Gamma_{\infty}$ is the the amount of baryons entrained as the fireball propagates through the stellar mantle.

Several analytic works have made estimates about the collimation angle and the Lorentz factor when the fireball breaks out the surface of the star (e.g., [32]). Nevertheless, the complexity inherent to the non-linear (magneto)hydrodynamic interaction of the fireball plasma with the stellar environment makes it unavoidable the use of numerical simulations. With these simulations we have been able to give preliminary answers to the following questions:

Collimation. The generated outflows are inertially or magnetically confined. In the first case, the collimator is the funnel within the progenitor while in the second case, the flow is self-collimated by its own magnetic field if it is strong enough. Rather independently on the initial conditions and on the inclusion of magnetic fields, the typical outflow half-opening angles, when the jet reaches the surface of the progenitor star, are $\theta_{\text {break }} \lesssim 5^{\circ}$. These small half-opening angles result from the recollimation of the outflow within the progenitor and they are independent on whether the boundary conditions are set to initiate the jet with much larger half-opening angles (e.g., $\theta_{0}=20^{\circ}$; [59]) or on whether the jet is generated by an energy release into a volume spanning a half-opening angle $\theta_{\mathrm{d}}=30^{\circ}$ much larger than $\theta_{\text {break }}$ [4]. In the course of their propagation through the progenitor the jets develop a non-homogeneous structure, transverse to the direction of motion, whose main features are an internal ultrarelativistic spine (where the Lorentz factor may reach $\Gamma_{\text {core }} \sim 30-50$ at jet breakout) within a half-opening angle of $<2^{\circ}$ laterally endowed by a moderately relativistic, hot shear layer $\left(\Gamma_{\text {shl }} \sim 5-10\right.$ ) extending up to $\theta_{\text {shl }}<20^{\circ}-30^{\circ}$ [5]. We point out that the transverse structure of the jet is nearly Gaussian both in simulations including magnetic fields (e.g., [31]) or not including them (e.g., [4]). However, a more accurate fit of the transverse structure of the jet, cannot be accommodated by a simple Gauss function [1].

Variability. All produced outflows are highly variable due to the generation of Kelvin-Helmholtz [4, 18], shear-driven [5] or pinch magnetohydrodynamic (MHD) instabilities [31]. Such extrinsic variability is independent on the (intrinsic) variability of the energy source and leads to the for- 

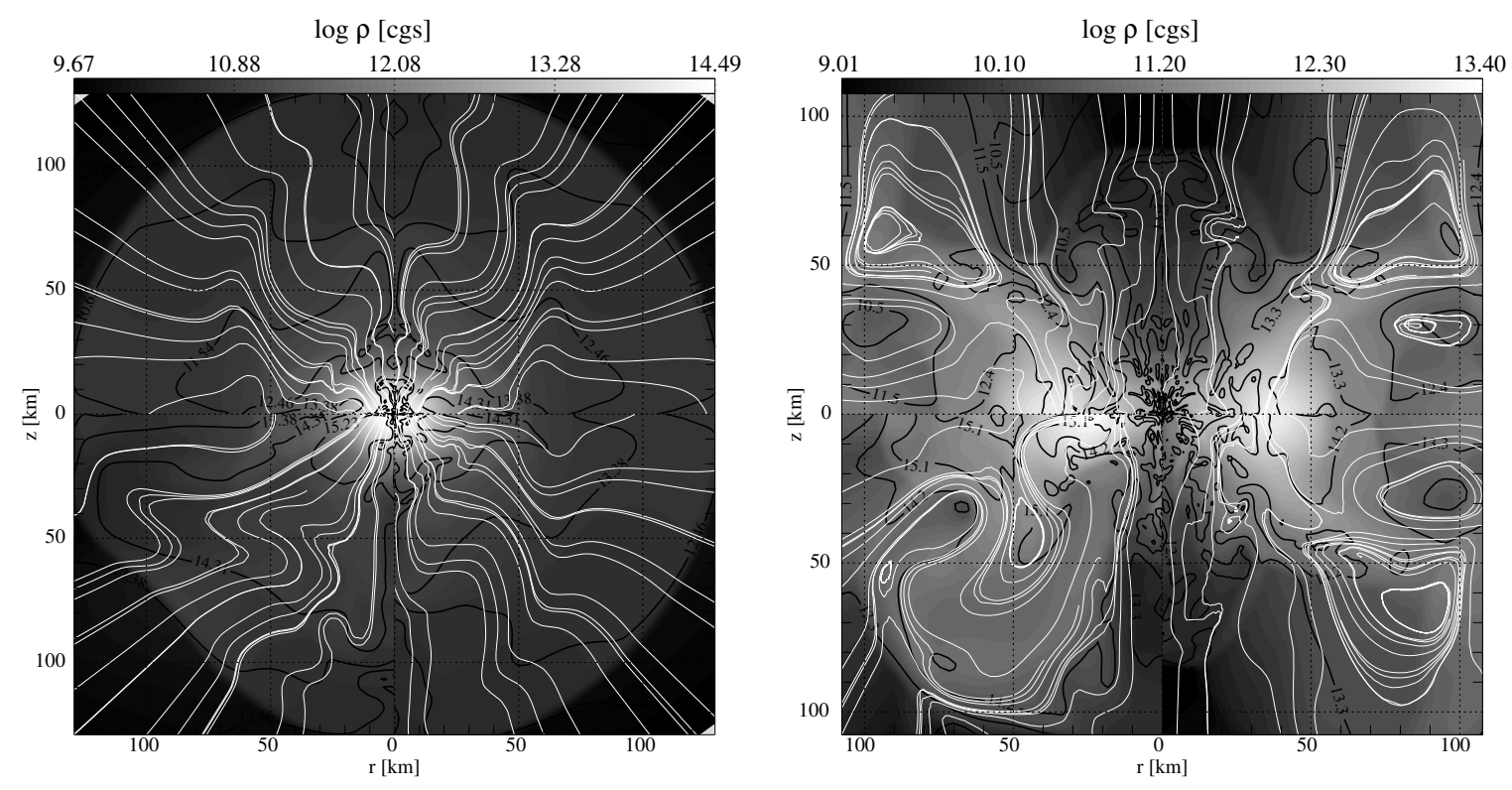

Figure 1: Logarithm of the density (gray-scale) with over-imposed magnetic field lines (white lines) and total magnetic field strength (black contours) corresponding to models collapsing stellar cores with a small initial rotational energy and rotating almost rigidly (left) and with a larger initial rotational energy and differentially rotating (right). Each panel displays the state of the stellar core after the bounce for four different initial magnetic field strengths $\left(B_{0}=10^{10} \mathrm{G}, 10^{11} \mathrm{G}, 10^{12} \mathrm{G}\right.$ and $10^{13} \mathrm{G}$ in the clockwise direction starting from the upper left corner). The figures correspond to models of Obergaulinger et al.[41].

mation of irregularities in the flow which are the seeds of internal shocks in the outflow. These internal shocks yield gamma-ray flares that produce the observed light curves (e.g., [48, 13, 33 35]). Except in cases in which the source may produce quasi-periodic variability (perhaps induced by precession or nutation modes of the accretion disc), the extrinsic variability might be indistinguishable form the intrinsic one. Numerical simulations of three-dimensional (3D) relativistic jets propagating through collapsar-like environments show that such jets are also stable [60] but it still remains unknown whether 3D relativistic, magnetohydrodynamic, collapsar-jets will also be stable along its whole trajectory.

Jet breakout. The jets generated are much lighter than their baryon reach environments. Thus, they propagate through the collapsar at moderate speeds $(\sim 0.3 c)$ and fill up thick cigar-shaped cavities or cocoons of shocked matter that also propagates along with the beam of the jet and that, eventually may break the surface of the collapsar. Since in the cocoon a few $10^{50} \mathrm{erg}$ may be stored as the jet drills its way through the star, it has been proposed that its eruption through the collapsar surface could yield a number of $\gamma$-ray/X-ray/UV-transients [47]. Indeed, it has been proposed that GRBs are but one observable phenomenon accompanying black hole birth and other possibilities may arise depending on the observer's viewing angle with respect to the propagation of the ultrarelativistic jet [58]. Thus, in a sort of unification theory for high-energy transients, one may see progressively softer events ranging from GRBs (when the jet emergence is seen almost head on) to UV flashes (when the jet eruption is seen at relatively large polar angles) and accounting for X-ray rich GRBs (XRR-GRBs) and X-ray flashes (XRF) at intermediate angles. The jet emer- 
gence through the stellar surface and its interaction with the stellar wind (which likely happens during the late stages of the evolution of massive stars) could lead to some precursor activity [29]. Furthermore, $v$-powered jets are very hot at breakout ( $\sim 80 \%$ of the total energy is stored in the form of thermal energy) which implies that jets can still experience an additional acceleration by conversion of thermal into kinetic energy, even if the energy source has ceased its activity.

\subsection{IGRBs produced in collapsars: MHD- or neutrino-powered jets?}

From a aesthetic point of view, it is beautiful if any invoked jet powering mechanism explains not only events with relatively small Lorentz factors $\Gamma \sim 100$ but also the occurrence of events with very large inferred values of $\Gamma \sim 500$ [27] or even $\Gamma \sim 1000$ as suggested by models for some GRBs [55]. However, there are no fundamental reasons to argue in favour of a unique and universal mechanism to extract the energy stored in the progenitor.

Purely hydrodynamic, $v$-powered jets in collapsars of type-I seem to be able only to produce more moderate terminal values of the bulk Lorentz factor ( $\Gamma \sim 100-400$; e.g., $[4,59])$, even if there is a further acceleration of the forward shock as a result of an appropriate density gradient in the medium surrounding the progenitor [4], unless the density in the funnel around the rotation axis is very small [32]. In collapsars of type-II the mass accretion rate $\left(\dot{M} \sim 10^{-3}-10^{-2} \mathrm{M}_{\odot} \mathrm{s}^{-1}\right)$ is insufficient to produce a $v$-powered jet, but it may suffice to generate MHD-powered jets whose observational signature might be weak, poorly collimated X-ray/UV transients or very long $(\sim$ $100-1000 \mathrm{~s})$ GRBs of low $E_{\text {iso }}$ and small $\Gamma$ if the progenitor star is able to loose its Hydrogen envelope [29].

From extrapolations of the numerical results of axisymmetric jets generated electromagnetically [31] very large values of the asymptotic Lorentz factor of the outflow $\left(\Gamma_{\infty} \sim 1000\right)$ can be attained. Thus, it seems rather plausible that MHD mechanisms have to be employed to generate jets with $\Gamma \sim 500-1000[30,31]$ and, invoking the existence of a universal energy extraction mechanism, one may also argue that also jets with much more moderate terminal Lorentz factors are also MHD-generated. Nevertheless, these arguments have a number of issues:

1.- The estimates of the terminal Lorentz factor of MHD-generated jets are based on axisymmetric models. The 3D-stability of relativistic magnetized jets is still a matter of debate which needs much more complex numerical simulations than the ones produced so far. Should 3D-MHD jets be unstable, should the terminal Lorentz factor not reliably be calculated with the state-of-theart axisymmetric, general relativistic, MHD (GRMHD) simulations up to date [31].

2.- GRMHD initial models of accreting BH systems consist on more or less realistic matter distributions over which an assumed poloidal field is imposed, i.e., they are not the result of a consistent magneto-rotational core collapse (e.g., [36, 37, 31]). The use of poloidal fields is triggered by the fact that purely toroidal field configurations do not yield the production of bipolar jets [14]. On the other hand, the initial magnetic field strengths are assumed, not consistently computed from the core collapse of massive stars. Typically, initial field strengths as large as $B \sim 10^{15}-10^{16} \mathrm{G}$ are used. Such large values of $B$ in combination with maximal values of the dimensionless angular momentum of the $\mathrm{BH}\left(j_{\mathrm{BH}} \sim 1\right)$ are necessary in order to efficiently extract energy via Blandford-Znajek (BZ) mechanism, because the BZ-power scales rather sensitive with $B$ as $\dot{E}_{\mathrm{BZ}} \sim 10^{50} j_{\mathrm{BH}}^{2}\left(M_{\mathrm{BH}} / 3 \mathrm{M}_{\odot}\right)^{2}\left(B / 10^{15} \mathrm{G}\right)^{2} \mathrm{erg} \mathrm{s}^{-1}[25]$. 
3.- When numerical simulations of the magnetorotational core collapse of massive stars are performed (see, e.g., [41, 42], and references therein), bipolar outflows as well as thick toroidal structures surrounding a central, low density funnel in the collapsed core are only generated for initial magnetic field strengths $B_{0} \gtrsim 10^{12} \mathrm{G}$ and for rather fast ${ }^{2}$ and differentially rotating stellar cores (Fig. 1 right). Such initial field strengths and angular velocities are well beyond the ones predicted by the state-of-the-art calculations of rotating massive stars [22]. These initially strongly magnetized models likely develop collapsed cores, with a mass of $M_{\mathrm{c}} \sim 1 \mathrm{M}_{\odot}$ and a specific angular momentum $J_{\mathrm{c}} \sim 10^{16} \mathrm{~cm}^{2} \mathrm{~s}^{-1}$, and may form a rapidly rotating $\mathrm{BH}$ with $j_{\mathrm{BH}} \sim 1$. Furthermore, the winding up of the initial poloidal field leads to maximal field strengths $B_{\max } \sim 10^{15} \mathrm{G}$, being the field predominantly toroidal $B_{\text {toroidal }} / B_{\text {poloidal }} \sim 1-10$. On the other hand, initially rigid, more moderately rotating cores do not yield tori around low-density funnels, do not produce bipolar jets and the maximum field strengths are $\sim 5 \times 10^{14} \mathrm{G}$ (Fig. 1 left). Considering that the collapsed cores are smaller $\left(M_{\mathrm{c}} \sim 0.75 \mathrm{M}_{\odot}\right)$ and slowly rotating $\left(J_{\mathrm{c}} \sim 2 \times 10^{15} \mathrm{~cm}^{2} \mathrm{~s}^{-1}\right)$ than in the previous case one may expect that the newly born $\mathrm{BH}$ resulting from the posterior evolution of these kind of cores will not be a maximally rotating but, instead, they will form BHs with more moderate $j_{\mathrm{BH}} \sim 0.6$. Thereby, the conclusion that seems to emerge from detailed numerical simulations of Obergaulinger et al.[41, 42] is that if the initial magnetic field strength and rotational energy is as small as predicted by the most detailed stellar evolution models, in the collapsed do not hold the appropriate conditions $\left(B \gtrsim 10^{15} \mathrm{G}, j_{\mathrm{BH}} \sim 1\right.$ ) to efficiently extract energy via BZ-mechanism and, conversely, unrealistically large initial magnetic fields and rotational energies need to be invoked to expect a BZ-like mechanism to operate efficiently. However, we have to be cautious in order not to extract too far reaching conclusions from the previously mentioned numerical work. It remains true that the results of the simulations of Obergaulinger et al. are handicapped because they do not include general relativity, because the numerical resolution is still small to capture all the relevant magneto-rotationally unstable modes and, because they are restricted to axisymmetric models.

From the above points, one may infer that the dynamical relevance of the magnetic field in the process of energy extraction from the central source will depend on fine details of the magnetorotational collapse of the collapsar core. On the other hand, the process of $v \bar{v}$-annihilation as the primary source of energy that fuels an ultrarelativistic fireball also needs of a more careful study in order to know how much energy such a process may release in the progenitor system and how such an amount of energy depends on the physical conditions of the progenitor.

A step towards such goal is the work of Birkl et al. [9], which contributes to better understand how the energy deposition rate due to the process of $v \bar{v}$-annihilation $\left(\dot{E}_{v \bar{v}}\right)$ depends on general relativistic (GR) effects and on different neutrinosphere geometries in hyperaccreting stellar-mass BH systems. Birkl et al. consider two families of neutrinospheres. On the one side, idealized geometries such as thin disks, tori, and spheres are studied. On the other side, more realistic models are constructed as non-selfgravitating equilibrium matter distributions for varied BH rotation. Independent of whether GR effects are included, considering the same values of temperature and surface area for an isothermal neutrinosphere, thin disk models yield the highest energy deposition rates by $v \bar{v}$-annihilation, while spherical neutrinospheres lead to the lowest ones. Considering isothermal neutrinospheres with the same temperature and surface area, it turns out that compared to Newto-

\footnotetext{
${ }^{2}$ The rotational energy being as large as $\sim 4 \%$ of the gravitational energy.
} 
nian calculations, GR effects increase the annihilation rate measured by an observer at infinity by a factor of 2 when the neutrinosphere is a disk (in agreement with the previous works; [8]). However, in case of a torus and a sphere the influence of GR effects is globally only $\sim 25 \%$, although locally, particularly in the vicinity of the rotation axis of the system, it can be significantly larger. Focusing on the dependence of the energy deposition rate on the value of $j_{\mathrm{BH}}$, it is found that increasing it from 0 to 1 enhances the energy deposition rate measured by an observer at infinity by roughly a factor of 2 due to the change of the inner radius of the neutrinosphere. Furthermore, although the absolute values of the energy deposition rate have to be taken with care (because of the steady state approximation used and the need of more realistic models for the accretion disk; see [9], for accretion disks of mass similar to the one expected in the collapsar model $\left(M_{\text {disk }} \lesssim 0.01 \mathrm{M}_{\odot}\right)$ typically, $\dot{E}_{v \bar{v}} \sim 10^{50}-10^{51} \mathrm{erg} \mathrm{s}^{-1}$. Even if only a small fraction $(\sim 10 \%)$ of that energy were used to boost a polar outflow, there is fair chance for neutrinos to be the dominant energy source of the fireball (at least, in some cases when the magnetic field is not too large). The most likely scenario that can be devised is that both mechanisms (MHD and neutrino energy release) might be operating simultaneously. Indeed, for MHD-produced jets, neutrinos will play a fundamental role in the pair-loading of the jet (e.g., [26]) while, for $v$-powered jets, the magnetic field may be important to collimate the thermally generated outflow. Which of the two energy deposition mechanisms dominates in every single GRB will depend on the exact conditions in the precollapse progenitor.

\section{Outflows emerging from progenitors of short GRBs}

Nowadays, it is commonly believed that short GRBs (sGRBs) are generated after the merger of a system compact binaries formed by either two neutron stars (NSs) or a neutron star and a BH $[44,19,15,38]$. The remnant left by the merger consists of a newly born BH black hole girded by a thick gas torus from which it swallows matter at a hypercritical rate. In such situation radiation is advected inward with the accretion flow and the cooling is dominated by the emission of neutrinos [46]. As in the case of progenitors of lGRBs, these neutrinos might either be the primary energy source blowing a fireball of $\mathrm{e}^{+} \mathrm{e}^{-}$pairs and photons or to act as mediator in hydromagnetic or electromagnetic energy extraction mechanisms (see Sect. 2) to pair-load the Poynting dominated outflow. A fundamental difference with respect to the collapsar model is that the accretion thick disk cannot be continuously refilled from a surrounding matter reservoir (the stellar matter in case of a collapsar) and, therefore, the duration of the produced outflows is, in part (see [6]) roughly limited by the time during needed by the black hole to engulf most of the matter of the accretion disk, namely, a few $100 \mathrm{~ms}$. This limit on the time scale, set by the ON time $t_{\mathrm{ce}}$ of the source, holds for both $v$-powered jets and for MHD-generated outflows. In the first case, the neutrino luminosity fades as the mass of the disk decreases and, thereby, there will be a critical torus mass below which a plasma outflow cannot be sustained. In the second case, for the same reason, there will not be sufficient neutrinos that pair-load the Poynting dominated jet after a sizable fraction of the torus has been accreted.

Although it seems likely that releasing a few $10^{49} \mathrm{erg}$ above the poles of a stellar mass BH in a region of nearly vacuum may yield an ultrarelativistic outflow, numerical simulations are needed to attempt to answer questions about the collimation mechanism of the polar outflow, the opening angle of the ultrarelativistic ejecta, the asymptotic Lorentz factors that can be attained, the internal 
structure of the outflow, the duration of a possible GRB event, and the isotropic equivalent energy which an observer would infer by assuming the source to expand isotropically. These questions may at most be guessed, but they cannot be reliably answered on grounds of merger models and a consideration of their energy release by neutrino emission and the subsequent conversion of some of this energy by $v \bar{v}$-annihilation to $\mathrm{e}^{+} \mathrm{e}$-pairs $[53,24,50,51,9]$. Only self-consistently timedependent (magneto)hydrodynamic modeling may give us some insight on the former questions. The reason being that the relativistic outflow develops in a complex interaction with the accretion torus, cleaning its own axial funnel such that later energy deposition encounters a much reduced baryon pollution.

Some keys to answer the questions mentioned in the previous paragraph have been very recently revealed by time-dependent numerical simulations in which the main results are:

Collimation. The generated outflows which may yield GRB signatures are either collimated by the accretion disk [6,7] or self-collimated by the magnetic field [31], depending on whether the jet is initiated thermally or magnetically, respectively. The typical outflow half-opening angles are $\sim 3^{\circ}-25^{\circ}$ and, as in the case of jets produced in collapsars, the baryon-poor outflows display a transverse structure. This structure shows a central core which spans a half opening angle $\theta_{\text {core }}<$ $3^{o}-12^{\circ}$ where $\Gamma_{\text {core }}>100$ flanked laterally by a layer, extending up to $\theta_{\text {shl }} \sim 25^{\circ}$ where the Lorentz factor smoothly decays to moderately relativistic values and where a sizable fraction of the total energy is stored. This layer is rather hot in thermally initiated outflows and has the potential of accelerating even after the energy release by the central engine has ceased. Similar to the jets produced in collapsar environments the transverse structure of the Lorentz factor could be roughly fit by Gaussian profiles. However, somewhat more complicated functions are required to provide more accurate fits [6].

Variability. Even injecting energy close to the BH even horizon at constant rates, the produced outflows are highly variable. The interaction of the newborn fireball with the accretion torus yields the growth of Kelvin-Helmholtz [6] instabilities. The variability in case of MHD jets is imprinted by pinch instabilities [31]. Up to date only axisymmetric models have been computed. All of which seem to be stable or marginally stable. It is not yet numerically verified whether $3 \mathrm{D}$ jets emerging from hyperaccreting BHs are stable.

Influence of the environment. Mergers of compact objects may take usually place in the intergalactic medium or in the outer skirts of their host galaxies. Thus, the environment of the merger may have a very low density. However, in the course of the merger, after the first contact of the two compact objects, there is an ejection of matter, which is larger close to the orbital plane. Such matter forms a cool baryon reach environment with a mass $M_{\text {halo }}<$ a few $10^{-2} \mathrm{M}_{\odot}[52,43]$. The exact amount of mass ejected sensitively depends on whether the two compact objects are both NSs or one of them is a $\mathrm{BH}$, on the initial mass ratio between the two, etc. Thereby, when the central $\mathrm{BH}$ forms, the newly born system may, in some cases, be embedded into a relatively high density halo. Mergers in low density environments may fuel ultrarelativistic outflows with the potential to produce normal sGRBs, while in case that the merger occurs in high density media, the observational signature is not a sGRB but, most likely, a flash in the soft X-ray or UV bands [6]. In the latter case, the resulting event might only be observable if it happens very close to the Milky Way. The fact that depending on the environmental density an SGRB can be produced or not has the direct implication that not every merger may yield a sGRB. This fact has to be considered when 
making estimates about the true rates of sGRBs and compared with the rates of NS+NS mergers (e.g., [20]).

Asymptotic Lorentz factor. The saturation value of the outflow Lorentz factor, $\Gamma_{\infty}$, is difficult to estimate on the basis of numerical simulations that cover only the initial fraction of a second in the evolution of an ultrarelativistic outflow generated in a hyperaccreting BH. Despite this difficulties rough estimates can be made. For instance, in case that the outflows are thermally generated [6], there is a clear trend to produce much higher values of $\Gamma_{\infty}$ for sGRBs $\left(\Gamma_{\infty} \gtrsim 500-1000\right)$ than for lGRBs $\left(\Gamma_{\infty} \sim 100\right)$. The reason for the difference resides on the much smaller density of the environment of the merger (even accounting for the mass ejected from the compact objects after the first contact; see above) as compared with the baryon-polluted environment that a relativistic jet finds inside a collapsing massive star. It has been speculated that this difference in Lorentz factor might be the reason for the paucity of soft sGRBs [23]. The former trend seems not to be followed by MHD-generated outflows [31]. The most likely reason being that McKinney's simulations are set to be scale free, while there should be a big difference between the environments of mergers of compact objects and the interior of collapsars. Probably, such a difference cannot be accommodated easily with simple scale-free power-laws for the distribution of the physical variables. Furthermore, the same concept of asymptotic Lorentz factor might be misleading since different regions of the ejected flow might reach distinct asymptotic values of the Lorentz factor. Indeed, this should be the case if the flow is stratified in the direction perpendicular to the flow velocity. Current numerical simulations $[6,7,39,31,40]$ show that the development of a velocity gradient in the direction normal to the radial direction is a robust numerical result. Thus, we should expect to find a variety of terminal Lorentz factors depending on the precise region of the ejecta we consider.

Duration of the events. As pointed out by Aloy et al. (2005) and Janka et al. (2006), the "shells" ejected by the central engine, accelerate much faster in the leading part of the outflow than the shells in its lagging part. The rear shells therefore need a longer time to reach velocities $v \simeq c$. This differential acceleration at early and late times of the relativistic jet leads to a stretching of the overall radial length of the outflow, $\Delta$, relative to $t_{\mathrm{ce}}$ times the speed of light $c, \Delta>c t_{\mathrm{ce}}$. This stretching has the important consequence that the overall observable duration of the GRB (in the source frame), $T=t_{\Delta}=\Delta / c$, may be a factor of 10 or more longer than $t_{\mathrm{ce}}$, even when the GRB is produced by internal shocks.

\section{Summary}

The numerical modelling of progenitors of GRBs has allowed us to gain some insight into a number of important issues related with the nature of the outflows produced by these systems. First, it has allowed us to verify that some (but probably not all) collapsars can yield collimated relativistic outflows that turn into lGRBs at large distances from the source. Likewise, only a fraction of the mergers of compact objects may yield sGRBs. Second, the numerical modeling has given us information about the collimation mechanism and typical outflow opening angles. Third, it has shown that the outflow is heterogeneous both along the direction of propagation and transverse to it, even if the central engine releases energy at a constant rate. Fourth, it seems rather plausible that some lGRBs with very high Lorentz factor $(\Gamma>>100)$ need of a MHD jet-formation mechanism. On the other hand, some other lGRBs with more moderate Lorentz factors $(\Gamma \sim 100)$ 
can be explained by $v$-powered jets, particularly if $t_{\mathrm{ce}}<10 \mathrm{~s}$. Fifth, MHD- and $v$-mechanisms may work simultaneously. Therefore, it is likely that, in some cases MHD processes dominate the jet generation while in others neutrinos may be the dominant energy source.

The numerical modeling done so far is still insufficient. In order to start from consistent initial models, the state-of-the-art numerical codes must incorporate, at least, the effects of strong gravity, magnetic fields and a detailed neutrino transport. In the near future we may see how all these elements are included in more realistic numerical experiments that will deepen our understanding on how ultrarelativistic outflows are produced in progenitors of GRBs.

\section{Acknowledgements}

MAA is a Ramón y Cajal Fellow of the Spanish Ministry of Education and Science. He also acknowledges partial support from the Spanish Ministry of Education and Science (AYA2004-08067C03-C01, AYA2007-67626-C03-01). PM is at the University of Valencia with a European Union Marie Curie Incoming International Fellowship (MEIF-CT-2005-021603). The authors thankfully acknowledge the computer resources, technical expertise and assistance provided by the Barcelona Supercomputing Center - Centro Nacional de Supercomputación.

\section{References}

[1] M. A. Aloy, Cataclysmic progenitors of gamma-ray bursts, (2001) in Highlights of Spanish Astrophysics II, p. 33

[2] M. A. Aloy, J. M. Ibáñez, J. M. Martí, E. Müller, GENESIS: A High-Resolution Code for Three-dimensional Relativistic Hydrodynamics, ApJS (1999) 122, 151

[3] M. A. Aloy, J. A. Pons, J. M. Ibañez, An efficient implementation of flux formulae in multidimensional relativistic hydrodynamical codes, Comp. Phys. Comm. (1999) 120, 115

[4] M. A. Aloy, E. Müller, J. M. Ibañez, J. M. Martí, A. MacFayden, Relativistic jets from collapsars, ApJ (2000) 531, L119

[5] M. A. Aloy, J.-M. Ibáñez, J. A. Miralles, V. Urpin, Stability analysis of relativistic jets from collapsars and its implications on the short-term variability of gamma-ray bursts $A \& A$ (2002) 396, 693

[6] M. A. Aloy, H.-Th. Janka, E. Müller, Relativistic outflows from remnants of compact object mergers and their viability for short gamma-ray bursts, $A \& A$ (2005) 436, 273

[7] M. A. Aloy, L. Rezzolla, A Powerful Hydrodynamic Booster for Relativistic Jets, ApJ (2006), 640, L115

[8] K. Asano, T. Fukuyama, Relativistic Effects on Neutrino Pair Annihilation above a Kerr Black Hole with the Accretion Disk, ApJ (2001) 546, 1019

[9] R. Birkl, M. A. Aloy, H. -Th. Janka, E. Müller, Neutrino pair annihilation near accreting, stellar-mass black holes, A\&A (2007) 463, 51

[10] R. D. Blandford, R. L. Znajek, Electromagnetic extraction of energy from Kerr black holes, MNRAS (1977) 179, 433

[11] R. D. Blandford, D. G. Payne, Hydromagnetic flows from accretion discs and the production of radio jets, MNRAS (1982) 199, 983 
[12] G. Cavallo, M. J. Rees, A qualitative study of cosmic fireballs and gamma-ray bursts, MNRAS (1978) 183, 359

[13] F. Daigne, R. Mochkovitch, Gamma-ray bursts from internal shocks in a relativistic wind: temporal and spectral properties, MNRAS (1998) 296, 275

[14] J.-P. De Villiers, J. F. Hawley, J. H. Krolik, S. Hirose, Magnetically Driven Accretion in the Kerr Metric. III. Unbound Outflows ApJ (2005) 620, 878

[15] D. Eichler, M. Livio, T. Piran, D. N. Schramm, Nucleosynthesis, neutrino bursts and gamma-rays from coalescing neutron stars, Nature (1989) 340, 126

[16] D. A. Frail, S. R. Kulkarni, S. R. Nicastro, M. Feroci, G. B.Taylor, The radio afterglow from the $\gamma$-ray burst of 8 May 1997, Nature (1997) 389, 261

[17] D. Giannios, P. Mimica, M. A. Aloy, On the existence of a reverse shock in magnetized GRB ejecta, $A \& A$ (2007) (in press); (arXiV:0710.1980)

[18] E. A. Gómez, P. E. Hardee, Collapsar Jet Stability at Breakout (2004), AIP Conf. Proc., 727, 278

[19] J. Goodman, Are gamma-ray bursts optically thick?, ApJL (1986) 308, L47

[20] D. Guetta, T. Piran, The luminosity and redshift distributions of short-duration GRBs, ApJ (2005) 435, 421

[21] F. A. Harrison, et al., Optical and Radio Observations of the Afterglow from GRB 990510: Evidence for a Jet, ApJL (1999) 523, L121

[22] A. Heger, S. E. Woosley, H. C. Spruit, Presupernova Evolution of Differentially Rotating Massive Stars Including Magnetic Fields, ApJ (2005) 626, 350

[23] H. -Th. Janka, M. A. Aloy, P. A. Mazzali, E. Pian, Off-Axis Properties of Short Gamma-Ray Bursts, ApJ (2006) 645, 1305

[24] H. -Th. Janka, Th. Eberl, M. Ruffert, C. L. Fryer, Black Hole-Neutron Star Mergers as Central Engines of Gamma-Ray Bursts, ApJ (1999) 527, L39

[25] H. K. Lee, R. A. M. J. Wijers, G. E. Brown, The Blandford-Znajek process as a central engine for a gamma-ray burst, Phys. Rep. (2000), 325, 83

[26] A. Levinson, D. Eichler, Baryon Purity in Cosmological Gamma-Ray Bursts as a Manifestation of Event Horizons, ApJ (1993) 418, 386

[27] Y. Lithwick, R. Sari, Lower Limits on Lorentz Factors in Gamma-Ray Bursts , ApJ (2001) 555, 540

[28] A. I. MacFadyen, S. E. Woosley, Collapsars: Gamma-Ray Bursts and Explosions in "Failed Supernovae”, ApJ (1999) 524, 262

[29] A. I. MacFadyen, S. E. Woosley, A. Heger, Supernovae, Jets, and Collapsars, ApJ (2001) 550, 410

[30] J. C. McKinney, Total and Jet Blandford-Znajek Power in the Presence of an Accretion Disk, ApJL (2005) 630, L5

[31] J. C. McKinney, General relativistic magnetohydrodynamic simulations of the jet formation and large-scale propagation from black hole accretion systems, MNRAS (2006) 368, 1561

[32] P. Mészáros, M. J. Rees, Collapsar Jets, Bubbles, and Fe Lines, ApJL (2001) 556, L37

[33] P. Mimica, M. A. Aloy, E. Müller, W. Brinkmann, Synthetic X-ray light curves of BL Lacs from relativistic hydrodynamic simulations, $A \& A$ (2004) 418, 947 
[34] P. Mimica, M. A. Aloy, E. Müller, W. Brinkmann, Which physical parameters can be inferred from the emission variability of relativistic jets?, A\&A (2005) 451, 103

[35] P. Mimica, M. A. Aloy, E. Müller, Internal shocks in relativistic outflows: collisions of relativistic shells, A\&A (2007) 466, 93

[36] Y. Mizuno, S. Yamada, S. Koide, K. Shibata, General Relativistic Magnetohydrodynamic Simulations of Collapsars, ApJ (2004a) 606, 395

[37] Y. Mizuno, S. Yamada, S. Koide, K. Shibata, , ApJ (2004b) 615, 389

[38] R. Mochkovitch, M. Hernanz, J. Isern, X. Martin, Gamma-ray bursts as collimated jets from neutron star/black hole mergers, Nature (1993) 361, 236

[39] B. J. Morsony, D. Lazzati, M. C. Begelman, Temporal and Angular Properties of Gamma-Ray Burst Jets Emerging from Massive Stars, ApJ (2007) 665, 569

[40] S. Nagataki, R. Takahashi, A. Mizuta, T. Takiwaki, Numerical Study of Gamma-Ray Burst Jet Formation in Collapsars, ApJ (2007) 659, 512

[41] M. Obergaulinger, M. A. Aloy, E. Müller, Axisymmetric simulations of magneto-rotational core collapse: dynamics and gravitational wave signal, ApJ (2006a) 450, 1107

[42] M. Obergaulinger, M. A. Aloy, H. Dimmelmeier, E. Müller, Axisymmetric simulations of magnetorotational core collapse: approximate inclusion of general relativistic effects, ApJ (2006b) 457, 209

[43] R. Oechslin, H. -Th. Janka, Torus formation in neutron star mergers and well-localized short gamma-ray bursts, MNRAS (2006) 368, 1489

[44] B. Paczyński, Gamma-ray bursters at cosmological distances, ApJ (1986) 308, L43

[45] Ph. Podsiadlowski, P. A. Mazzali, K. Nomoto, D. Lazzati, E. Capellaro, The Rates of Hypernovae and Gamma-Ray Bursts: Implications for Their Progenitors, ApJL (2004) 607, L17

[46] R. Popham, S. E. Woosley, C. Fryer, Hyperaccreting Black Holes and Gamma-Ray Bursts, ApJ (1999) 518, 356

[47] E. Ramirez-Ruiz, A. Celotti, M. J. Rees, Events in the life of a cocoon surrounding a light, collapsar jet, MNRAS (2002) 337, 1349

[48] M. J. Rees, P. Mészáros, Unsteady outflow models for cosmological gamma-ray bursts, ApJ (1994) 430, L93

[49] J. E. Rhoads, The Dynamics and Light Curves of Beamed Gamma-Ray Burst Afterglows, ApJ (1999) 525, 737

[50] S. Rosswog, E. Ramirez-Ruiz, Jets, winds and bursts from coalescing neutron stars, MNRAS (2002) 336, L7

[51] S. Rosswog, E. Ramirez-Ruiz, M. B. Davies, High-resolution calculations of merging neutron stars III. Gamma-ray bursts, MNRAS (2003) 345, 1077

[52] M. Ruffert, H. -Th. Janka, Coalescing neutron stars - A step towards physical models. III. Improved numerics and different neutron star masses and spins, $A \& A$ (2001) 380, 544

[53] M. Ruffert, H. -Th. Janka, Gamma-ray bursts from accreting black holes in neutron star mergers, $A \& A$ (1999) 344, 573

[54] R. Sari, T. Piran, J. P. Halpern, Jets in Gamma-Ray Bursts, ApJL (1999) 519, L17 
[55] A. M. Soderberg, E. Ramirez-Ruiz, Flaring up: radio diagnostics of the kinematic, hydrodynamic and environmental properties of gamma-ray bursts, MNRAS (2003) 345, 854

[56] G. B. Taylor, D. A. Frail, E. Berger, S. R. Kulkarni, The Angular Size and Proper Motion of the Afterglow of GRB 030329, ApJL (2004) 609, L1

[57] S. W. Woosley, Gamma-ray bursts from stellar mass accretion disks around black holes, ApJ (1993) 405, 273

[58] S. E. Woosley, Gamma-ray bursts: The central engine (2000), AIP Conf. Proc. 526, 555

[59] W. Zhang, S. E. Woosley, A. I. MacFadyen, Relativistic Jets in Collapsars, ApJ (2003) 586, 356

[60] W. Zhang, S. E. Woosley, A. Heger, The Propagation and Eruption of Relativistic Jets from the Stellar Progenitors of Gamma-Ray Bursts, ApJ (2004) 608, 365 